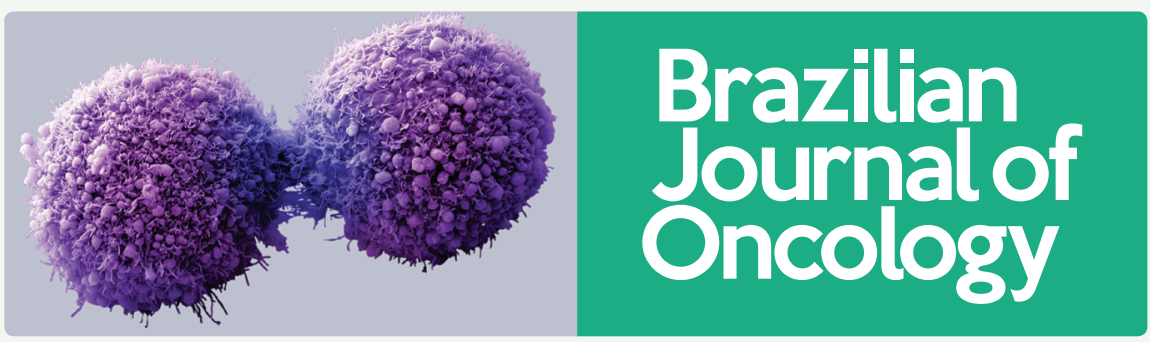

EDITORIAL

\title{
Improving the adjuvant treatment of HER2-positive breast cancer: APHINITY and ExteNET trials
}

\author{
Melhorando o tratamento adjuvante do câncer de mama HER2-positivo: Estudos \\ APHINITY e ExteNET
}

Noam Pondé1, Evandro de Azambuja ${ }^{1}$

The natural history of HER2-positive early breast was dramatically changed in 2005 by the results of 4 randomised phase III trials evaluating the combination of chemotherapy with adjuvant trastuzumab, the first drug in the anti-HER2 class. HERA, BCIRG-006, NCCTG 9831 and NSABP-B31 established 1 year of adjuvant trastuzumab as the standard of care, and long term follow-up results of these trials (8 to 11 years) have underscored the significant and prolonged improvement in outcomes patients derive from trastuzumab use. (1) Toxicity, mostly cardiac in nature, has proven to be low when patients are carefully selected and followed. In addition, most cardiac events are reversible with interruption of trastuzumab and prompt cardiac treatment.(2) Though the cost associated with adjuvant trastuzumab can be a concern, especially in developing countries, the development of biosimilar options will likely bring prices down - making trastuzumab a safe, effective and relatively affordable treatment standard.(3) Despite these improvements, HER2-positive patients still relapse in significant numbers. The 11 year follow-up results of HERA show that approximately $30 \%$ of patients in the trastuzumab containing arms eventually experienced a relapse. ${ }^{(5)}$ After a new generation of anti-HER2 agents successfully improved outcomes and changed clinical practice in the advanced setting - including lapatinib, pertuzumab and T-DM1, it was only logical to hope they would be similarly effective in early disease. ${ }^{(6)}$ The first results of lapatinib and pertuzumab in the neoadjuvant setting showed significant improvement in pathologic complete response (pCR). NeoALTTO tested trastuzumab + lapatinib vs trastuzumab or lapatinib alone, first alone then with paclitaxel, followed by surgery and further chemotherapy and the same anti-HER2 therapy for a year. ${ }^{(7)}$ Results showed the superiority of the dual blockade regimen (with an absolute improvement in pCR of approximately 20\%). The NEOSPHERE study tested docetaxel + trastuzumab, docetaxel + pertuzumab or both monoclonal antibodies with or without docetaxel, also showing superior results with the dual blockade regimen (absolute improvement in pCR of approximately

1. Institut Jules Bordet, Brussels, Belgium.

Conflicts of interest: Both authors are active in the management of the APHINITY trial. Evandro de Azambuja is a member of the APHINITY Steering Committee and is the Chairperson of the KIARA trial, fully funded by a grant from Roche/Genentech. Evandro de Azambuja has received travel grants from Roche and fees for advisory boards.

Correspondence author: Evandro de Azambuja. Blvd de Waterloo, 121, 1000 Brussels, Belgium. Phone: +32 25417244

E-mail: evandro.azambuja@bordet.be.

Received on: January 28, 2018 | Accepted on: March 19, 2018

DOI: 10.26790/BJO20181447A188 
16\%). ${ }^{(8)}$ Both trials, however, failed to show an equivalent improvement in disease-free survival (DFS)/event-free survival (EFS), though it must be said that neither trial was adequately powered to show a significant improvement in long outcomes. To change standards in the adjuvant setting, larger phase III studies were needed, leading to the design of the dual blockade ALTTO and APHINITY trials. ${ }^{(10,11)}$ Additionally, despite negative results in advanced disease, the ExteNET trial testing an alternative strategy of extending anti-HER2 therapy to 2 years with neratinib after the completion of trastuzumab was also launched. ${ }^{(12,13)}$

The first evidence that the extent of success seen in the advanced setting would not be easily replicated were the results of the ALTTO trial, which randomised 8381 patients to 4 arms testing trastuzumab and lapatinib as single agents, in sequence (trastuzumab followed by lapatinib) or in combination (trastuzumab + lapatinib). Though the reasons for this trial proving statistically negative, despite some signal of efficacy, have been extensively debated in recent years, it brought an end to the development of lapatinib in the early setting. On the other hand, both APHINITY and ExteNET were positive trials - showing a significant improvement in invasive disease-free survival (IDFS), ultimately leading to approval by the FDA of both regimens tested in these trials.

APHINITY tested chemotherapy (either anthracycline-based or anthracycline-free) combined with trastuzumab or trastuzumab + pertuzumab for 1 year in 4805 patients. Results of the primary analysis show a statistically significant yet small absolute improvement in 3-year IDFS favouring the arm receiving dual blockade of 0.9\% (hazard ratio, 0.81; 95\% confidence interval $[\mathrm{Cl}], 0.66$ to 1.00; $\mathrm{p}=0.045)$, with absolute gains being somewhat better at 4 years (1.7\%). Subgroup analysis suggests that patients with node positive disease benefitted more than node negative patients (1.8\% absolute IDFs gain at 3-years), as well as a non-significant trend towards higher benefit in estrogen receptor negative (ER-negative) patients. The adverse event profile with the dual blockade regimen was overall similar to the trastuzumab single agent regimen, with the exception of a significant increase in the incidence of diarrhoea (Grade 3 or more $9.8 \%$ vs 3.7\%), though it is important to note that almost all cases occurred during the chemotherapy phase. (11) Primary cardiac events were numerically increased in the combination group (17 vs 8), however the overall numbers were very low, highlighting the safety of the combination regimen in a carefully selected patient population.

The ExteNET trial randomised 2840 patients to receive standard chemotherapy and trastuzumab regimen followed either by one additional year of neratinib or placebo. 5-year IDFS results show the relative superiority of the extended regimen with an absolute benefit of 2.5\% (HR 0.73, 95\% Cl 0.57-0.92, p=0.0083). Subgroup analysis suggest increased efficacy in patients with ER-positive tumours. The toxicity profile was, however, pronouncedly more intense in the extended treatment arm, notably with diarrhoea ( $40 \%$ vs $<1 \%)$, nausea ( $3 \%$ vs $<1 \%)$ and vomiting $(2 \%$ vs $<1 \%)$ grade 3 or more, which was reflected in reduced median dose intensity (82\% in neratinib arm vs 98\% placebo arm). ${ }^{(12)}$

Placing these results into the context of clinical practice, however, is likely to prove challenging for clinicians. Though both dual blockade and extended therapy proved to improve outcomes, these improvements are exceedingly small when compared to the absolute improvement in disease free survival (DFS) of $6.8 \%$ in the HERA trial. Therefore, most patients are still candidates for chemotherapy + 1 year trastuzumab and a clear example are patients falling into populations well represented in APT (T1, node negative patients with ER-positive disease) to whom paclitaxel + trastuzumab alone is a good option. ${ }^{(4)}$ For patients who are not candidates to this regimen, clinicians must carefully decide to either continue to use 1 year of trastuzumab alone, dual blockade with pertuzumab or extended therapy with neratinib. For high risk patients, especially those who are candidates for neoadjuvant therapy, node positive and/or ER-negative tumors, dual blockade with pertuzumab should be considered. Extended therapy with neratinib can be an option for patients at a high risk of relapse who did not receive neoadjuvant chemotherapy, especially if ER+. However, proactive management of diarrhoeas, as per the CONTROL (NCT02400476) trial, is needed to prevent low-adherence to such prolonged oral treatment. 
There is no doubt that progress was achieved in the HER2-positive breast cancer population, with at least three treatment options in 2018: 1 year of trastuzumab, dual blockade with trastuzumab and pertuzumab or neratinib after trastuzumab. However, a better understanding on which population benefits the most from each of these approaches is urgently needed to better fine-tune adjuvant treatment and can only be achieved through better trial design that integrate biomarker research and selective escalation strategies.

\section{REFERENCES}

1. Moja L, Tagliabue L, Balduzzi S, et al. Trastuzumab containing regimens for early breast cancer [Internet]. In: The Cochrane Collaboration, editor. Cochrane Database of Systematic Reviews. Chichester, UK: John Wiley \& Sons, Ltd; 2012 [cited 2017 Jul 19]. Available from: http://doi.wiley.com/10.1002/14651858.CD006243.pub2

2. Pondé NF, Lambertini M, de Azambuja E. Twenty years of anti-HER2 therapy-associated cardiotoxicity. ESMO Open. 2016;1(4):e000073.

3. Lambertini M, Pondé NF, Solinas C, de Azambuja E. Adjuvant trastuzumab: a 10-year overview of its benefit. Expert Rev Anticancer Ther. 2017;17(1):61-74

4. Tolaney SM, Barry WT, Dang CT, Yardley DA, Moy B, Marcom PK, et al. Adjuvant paclitaxel and trastuzumab for nodenegative, HER2-positive breast cancer. N Engl J Med. 2015;372(2):134-41.

5. Cameron D, Piccart-Gebhart MJ, Gelber RD, Procter M, Goldhirsch A, de Azambuja E, Castro G Jr, Untch M, Smith I, Gianni L, Baselga J, Al-Sakaff N, Lauer S, McFadden E, Leyland-Jones B, Bell R, Dowsett M, Jackisch C; Herceptin Adjuvant (HERA) Trial Study Team. 11 years' follow-up of trastuzumab after adjuvant chemotherapy in HER2 positive early breast cancer: final analysis of the HERceptin Adjuvant (HERA) trial. Lancet. 2017 ;389(10075):1195-205.

6. Giordano SH, Temin S, Kirshner JJ, Chandarlapaty S, Crews JR, Davidson NE,Esteva FJ, Gonzalez Angulo AM, Krop I, Levinson J, Lin NU, Modi S, Patt DA, Perez EA, Perlmutter J, Ramakrishna N, Winer EP; American Society of Clinical Oncology. Systemic therapy for patients with advanced human epidermal growth factor receptor 2-positive breastcancer: American Society of Clinical Oncology Clinical Practice Guideline. J Clin Oncol. 2014;32(19):2078-99.

7. de Azambuja E, Holmes AP, Piccart-Gebhart M, Holmes E, Di Cosimo S, Swaby RF, et al. Lapatinib with trastuzumab for HER2-positive early breast cancer (NeoALTTO): survival outcomes of a randomised, open-label, multicentre, phase 3 trial and their association with pathological complete response. Lancet Oncol. 2014;15(10):1137-46.

8. Gianni L, Pienkowski T, Im Y-H, Roman L, Tseng LM, Liu MC, et al. Efficacy and safety of neoadjuvant pertuzumab and trastuzumab in women with locally advanced, inflammatory, or early HER2-positive breast cancer (NeoSphere): a randomised multicentre, open-label, phase 2 trial. Lancet Oncol. 2012;13(1):25-32.

9. Gianni L, Pienkowski T, Im Y-H, Im YH, Tseng LM, Liu MC, et al. 5-year analysis of neoadjuvant pertuzumab and trastuzumab in patients with locally advanced, inflammatory, or early-stage HER2-positive breast cancer (NeoSphere): a multicentre, openlabel, phase 2 randomised trial. Lancet Oncol. 2016;17(6):791-800. 
10. Piccart-Gebhart M, Holmes E, Baselga J, de Azambuja E, Dueck AC, Viale G, et al. Adjuvant lapatinib and trastuzumab for early human epidermal growth factor receptor 2-positive breast cancer: results from the randomized phase iii adjuvant lapatinib and/or trastuzumab treatment optimization trial. J Clin Oncol Off J Am Soc Clin Oncol. 2016;34(10):1034-42.

11. von Minckwitz G, Procter M, de Azambuja E, Zardavas D, Benyunes M, Viale G, et al. Adjuvant pertuzumab and trastuzumab in early HER2-positive breast cancer. N Engl J Med. 2017;377(2):122-31.

12. Martin M, Holmes FA, Ejlertsen B, Delaloge S, Moy B, Iwata H, von Minckwitz G, Chia SKL, Mansi J, Barrios CH, Gnant M, Tomaševic Z, Denduluri N, Šeparovic R, Gokmen E, Bashford A, Ruiz Borrego M, Kim SB, Jakobsen EH, Ciceniene A, Inoue K, Overkamp F, Heijns JB, Armstrong AC, Link JS, Joy AA, Bryce R, Wong A, Moran S, Yao B, Xu F, Auerbach A, Buyse M, Chan A; ExteNET Study Group. Neratinib after trastuzumab-based adjuvant therapy in HER2-positive breast cancer (ExteNET): 5-year analysis of a randomised, double-blind, placebo-controlled, phase 3 trial. Lancet Oncol. 2017;18(12):1688-1700.

13. Awada A, Colomer R, Inoue K, Bondarenko I, Badwe RA, Demetriou G, et al. Neratinib plus paclitaxel vs trastuzumab plus paclitaxel in previously untreated metastatic ERBB2-Positive Breast Cancer: The NEfERT-T Randomized Clinical Trial. JAMA Oncol. 2016;2(12):1557. 